\title{
Research Paper: The Differences in Sensorimotor Rhythm Power During Performing In-Phase and Anti-Phase Patterns in Bimanual Coordination
}

\author{
Ebrahim Norouzi $^{1^{*}}$ (D), Mohammad Vaezmousavi ${ }^{2,3}$
}

1. Department of Motor Behavior and Sport Management, Faculty of Sport Sciences, Urmia University, Urmia, Iran. 2. Department of Physical Education, Faculty of Social and Cultural Sciences, Imam Hossein University, Tehran, Iran. 3. Institute of Social and Cultural Sciences, Imam Hossein University, Tehran, Iran.

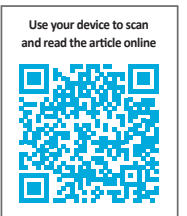

drtat on Norouzi E, Vaezmousavi M. The Differences in Sensorimotor Rhythm Power During Performing In-Phase and AntiPhase Patterns in Bimanual Coordination. Physical Treatments. 2018; 8(1):1-8. http://dx.doi.org/10.32598/ptj.8.1.1

doi : http://dx.doi.org/10.32598/ptj.8.1.1

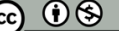

\section{Article info:}

Received: 10 Nov 2017

Accepted: 23 Feb 2018

Available Online: 01 Apr 2018

Keywords:

Neurofeedback,

Movement, Psychology,

Psychomotor

performance, Athletic

performance

\section{A B S T RA C T}

Purpose: The sensorimotor cortex oscillations (frequency ranging between 12 and $15 \mathrm{~Hz}$ ), commonly known as Sensorimotor Rhythm (SMR) has previously displayed a promising link between the performance of the visuomotor related to skill execution and part of psychology that is adaptive (e.g. the process linked attention which is automatic). This study examined the extent of SMR power in the execution of both in- and anti-phase patterns in bimanual coordination tasks at different speeds.

Methods: The present study used a quasi-experimental method. Study participants $(n=40$, aged: 19-24 years) were selected using convenience sampling method. Study participants were subjected to the 2 bimanual movements with the wrists speed levels ranging from slow to fast; while taking simultaneous records of the EEG. The neurofeedback consisted of SMR frequency of $12-15 \mathrm{~Hz}$ at $\mathrm{C} 3$ and $\mathrm{C} 4$. Data analysis consisted of descriptive statistics and 2-way repeated measures Analysis of Variance (ANOVA) using SPSS. Examination of post-hoc results of importance was conducted using Bonferroni correction paired comparisons. $\mathrm{P}=0.05$ was set as the significance level.

Results: The results suggested that SMR power was higher in anti-phase compared to the inphase model. In addition, the manipulation of bimanual speed affected the SMR power by increasing it in the anti-phase when the speed increased. However, the SMR power did not raise when the in-phase pattern was conducted.

Conclusion: Further attention is needed in the anti-phase model as it requires greater SMR power wave. Moreover, with increasing speed, the amount of SMR power can perform a better bimanual linear task.

\footnotetext{
* Corresponding Author:

Ebrahim Norouzi, $P h D$.

Address: Department of Motor Behavior and Sport Management, Faculty of Sport Sciences, Urmia University, Urmia, Iran

Phone: +98 (912) 9469087

E-mail: eb.norouzi@urmia.ac.ir
} 


\section{Highlights}

- Higher sensory-motor rhythm power is associated with better motor control.

- Specific higher sensory-motor rhythm improves bimanual coordination.

- The sensorimotor rhythm can enhance the motor control of people with motor disorders.

\section{Plain Language Summary}

Neurofeedback Training (NFT) is a method to treat pain. In addition, NFT has positive effects of on chronic pain, attention-deficit/hyperactivity disorder, enhancement of motor performance, and cognitive flexibility. In this paper, we demonstrate that bimanual coordination accuracy and consistency is more associated with higher Sensorimotor Rhythm (SMR), and particularly during the anti-phase of bimanual coordination. SMR neurofeedback training can be recommended as add-ons to standard care to improve motor control and reduce motor function impairment among patients with motor disorders. The current study adds to the literature in an important way indicating that higher SMR power has benefits for both in-phase and anti-phase bimanual coordination patterns.

\section{Introduction}

lectroencephalogram (EEG) is a powerful

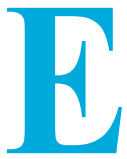
tool used for studying cortical excitability during motor performance $[1,2]$. Attention is essential in motor skills preparation, because attentional shift is important in elite athletes and competitive situations [3]. Sensorimotor Rhythm (SMR) is used to examine differences in cortical activities. It is also an indicator of attention [4]. Moreover, it is inversely associated with sensorimotor activity of the cortex. SMR training investigations indicate that people can increase the power of SMR to maintain accuracy and attention in performance [5]. Therefore, researchers should design neurofeedback trainings that can enhance the activity of SMR and to facilitate motor performance in individuals. SMR training increases the working memory capacity and the focus of attention, which improves the preparation of motor action [6] and elevates the mood [7].

Expert athletes show more particular cortex activities than novices during planning and performing movements [8]. For example, expert athletes show reduced verbal-analytical processes in left temporal regions [9] and increased SMR power at $\mathrm{Cz} 4$ [10]. Cheng et al. examined the SMR differences in novices and expert dart players. Their results demonstrated a higher SMR power before the throw in skilled players [4]. Therefore, the motor task can be performed more accurately by increasing the SMR power waves. Thus, the motor perception decreases following an increase in SMR activity, to develop focus and relaxation as a result of the diminishing processes of the somatosensory elements. Furthermore, Doppelmayr and Weber discovered that besides significantly increasing the SMR amplitude, the augmented SMR training positively affects the spatialrotation ability and decision-making reaction time [11] The present study aimed to examine whether various amounts of SMR power waves are required when performing bimanual coordination activities in either of the 2 opposite phases.

An appropriate performance of bimanual movements is constrained by essential principles of coordinative behaviors [12-16]. The relative phases of 00 and 1800 in the in-phase and anti-phase, create stable coordination patterns, respectively, in terms of the upper limb in the horizontal plane [14-16]. The in-phase coordination mode involves simultaneous activation of the homologous group of muscles by concurrent mirror symmetric movements away and towards the body through the body centerline $[14,17,18]$. Simultaneous movements from one side of the body to the other through the midline of the body produce iso-directional (parallel) patterns of movements in the natural space that generate the anti-phase coordination mode $[17,19,20]$. The in-phase coordination does not change after an increase in the frequency of movement, while the anti-phase coordination becomes unstable. An eventual spontaneous transition is achieved in the in-phase, if the increasing frequency is not opposed [12, 21, 22].

Kelso suggested a task in both fixed patterns including the 00-relative phase and 1800-relative phase, to study the movements of 2 limbs. Increasing the mo- 
tion speed significantly affects the anti-phase [12, 21]. However, the model lacks observable effects on the in-phase design. The anti-phase pattern variably corrupts, changes and reduces its precision, by the gradual increase of rate or motion $[13,23]$. This finding suggests that the in-phase does not readily change, compared to the opposite patterns $[17,24]$. In other words, the required attention process is more extensive in the anti-phase than in-phase patterns. We employed a task in the bimanual coordination of the 00-phase and the 1800-phase models using movements of flexion and the extensions. The study participants used visual, proprioception or audition feedback loops to extensively control the limbs, in order to investigate the continuous nature of the bimanual actions.

EEG studies strongly suggest that cortical activity, related to the band-limited EEG analysis, demonstrate the cognitive processes with remarkable functionality and provide more information than wide EEG analysis $[25,26]$. Therefore, we examined the waves separately in the present study. However, the question raised based on the presented material is whether the SMR power differs between the anti-phase and in-phase patterns. Also which coordination patterns requires more SMR power, in case of any differences. In addition, we compared SMR power at different speeds of each model. This designed experiment also investigated the changes in the performance and stability of bimanual coordination models at varying movement speeds. This study also explained how they differ from each other with respect to the degrees of SMR power.

\section{Materials and Methods}

This was a quasi-experimental study with factorial design. The statistical population included all nonphysical education major students of Shahid Beheshti University. Study participants were selected using convenience sampling method. All participants were righthanded (according to E.H. Inventory; Oldfield) [27]. The samples aged between 19 and 24 years with the mean age of 21 years. They were included according to standardized self-report auditions, the Snellen chart test, and lack of any diagnosed neuromuscular, motor and/or sensory disorders. The health conditions of the participants were investigated using a questionnaire. These questionnaires were completed prior to obtaining the informed consent forms, in order to assess the subjects with regard to the inclusion criteria of the study. The findings of present study could be applicable for improving the motor performance in healthy young males by means of SMR neurofeedback training.

\section{Study procedure}

The ProComp Infiniti (2180 Belgrave Avenue, Montreal, QC H4A 2L8 Canada) encoder is a computerized biofeedback data acquisition device with 8 multi-modal channels for real-time recording. It has 8 pin sensor inputs and $2048 \mathrm{~s} / \mathrm{s}$ and $256 \mathrm{~s} / \mathrm{s}$ samples of 2 and 6 channels, respectively. The ProComp Infiniti encoder can objectively read a broad scope of clinical and physiological signs for observation and biofeedback. We reorganized functionality applying BioGraph software.

Only wrist movements of the flexion and extension inclinations from the centerline of the body were permitted. Linear potentiometers were symmetrically attached to the slides (Bourns Instruments, Riverside, CA). These actions provided data regarding the changing distances of the handle with a 20-s sequence. An 80486 microprocessor was employed to sample the data within the range of $150 \mathrm{~Hz}$. A National Instruments software; LabWindows started and stopped at 20-s sequences while presenting the captured data of the position of the limb in a time frame. The position signals were rectified with asymmetrical (triangular) Bartlett filter. The velocity time signals were obtained using the impulse of localised points by the 2-point CDA (central difference algorithm). We also applied Bartlett software prior to further rectification. The formula adapted from Kelso $[12,21]$ and the smoothed time-series of position and velocity were applied to compute every component of the near continuous phase of the sequences.

$$
\phi R=\tan -\{(d X R / d t) / X R\})
$$

The right wrist phase at every selected XR provides the location of the right wrist with an adjusted scale to the interval $(-1,1)$ in every complete oscillation, while (dXR/ dt) provides its standardized velocity at each instance. We used the same formula for the left wrist calculations of velocity and position signals. The phase relating the 2 wrists was calculated by the following formula:

$$
\varphi=\phi R-\phi L
$$

The mean Absolute Error (AE) of the relative phase was a mirror image of deviation from the intended relative phase $\left(0^{\circ}\right.$ for the in-phase mode and $180^{\circ}$ for the anti-phase mode) (precision coordination). An auditory metronome (NCH Swift Sound Tone Generator, version 2.01) provided information on the changing pace for the increasing speed of bimanual activity [28]. 
Initially, the resting EEG of the subjects were recorded. This consisted of a shifted condition of simultaneous closing and opening of one eye. The EEGs were recorded at 2 electrode sites $(\mathrm{C} 3, \mathrm{C} 4)$ corresponding to the international 10-20 system [29]. At first, every site had an AI as the reference, then it was re-referred to linked offline ears. EEG data were collected and amplified using ProComp Infiniti system by BioGraph software. EEG signals were recorded online. BioGraph Infiniti software was installed on a Lenovo 8023 laptop. C3 and C4 for SMR power were the position of the electrode with the reference set on the right ear. The signal was changed, and the band was rectified to produce the 12 to $15 \mathrm{~Hz}$ SMR wave. Each participant was then oriented to the task.

A wrist extension and flexion in the left-right dimension task, required the participants to grasp 2 handles on a moving slide and move them horizontally. Each participant produced the 2 opposite phased patterns, while holding both handles. All study participants moved their hands continuously toward and then away from each other in a symmetrical manner to activate the homologous flexors and extensors wrist muscles, during the in-phase pattern. The hands moved in unison and in an iso-direction, during the anti-phase pattern. The homologous group of muscles contracted in an alternating manner in this phase. The subjects were then instructed to keep up with the pace of a metronome by conducting a complete displacement of the handle in a cycle of in-out-in time beats. The metronome facilitated the required frequency or speed gradually, from a 20-s frequency of $58 \mathrm{~B} / \mathrm{M}$. After the low-speed 20-second trial, a middle range frequency of $90 \mathrm{~B} / \mathrm{M}$ was paced in the same coordination activity, before the final subsequent $152 \mathrm{~B} / \mathrm{M}$ was used.

EEG sections with 6 amplitudes beyond $\pm 100 \mu \mathrm{V}$ were excluded from the analysis. Seven EEG data without any artifacts were taken. Hanning window (50\%) was applied to these segments and thereafter Fast Fourier Transform (FFT) was used to calculate mean power spectra in the SMR (12-15 Hz). The SMR power was computed using the 12 to $15 \mathrm{~Hz}$ averages from $\mathrm{C} 3$ and $\mathrm{C} 4$, before transforming the natural logs.

\section{Statistical analysis}

The obtained data were analyzed by descriptive statistics and 2-way repeated measures Analysis of Variance (ANOVA) ( 2 bimanual coordination patterns $\times 3$ speed), using SPSS. Examination of post-hoc results of importance was conducted using Bonferroni correction paired comparisons. $\mathrm{P}=0.05$ was set as the significance level.

\section{Results}

Two-way repeated measures ANOVA results for the SMR power (Figure 1) revealed the significant main effect for speed $\left(\mathrm{F}_{2}=1.35, \mathrm{P}=0.001\right)$. Also, the primary effect of bimanual pattern was significant $\left(\mathrm{F}_{1}=7.39\right.$, $\mathrm{P}=0.001)$. The interaction effect of speed and bimanual pattern was significant $\left(\mathrm{F}_{2}=1.27, \mathrm{P}=0.001\right)$ (Table 1). The Bonferroni was used for more analysis of the twoway interaction of significance for bimanual pattern $x$ speed. In anti-phase pattern, speed significantly affected all conditions $(\mathrm{P}=0.001)$. Through the pairwise comparisons, a major increase of SMR power were noted between slow versus medium pace $(\mathrm{P}=0.001)$, slow versus fast pace $(\mathrm{P}=0.001)$ and medium versus fast pace $(\mathrm{P}=0.001)$. These observations indicate that the increasing movement speed strongly influences the SMR power in the anti-phase coordination mode. In addition, pairwise comparisons between conditions reveals that the study participants produced in-phase movements with similar levels of SMR power during slow speed, average speed and fast speed (all $\mathrm{P}>0.4$ )

The results of two-way repeated measures ANOVA for the AE scores in the bimanual coordination mode (Figure 2) revealed a significant primary effect for bimanual patterns $\left(\mathrm{F}_{1}=7.39, \mathrm{P}=0.001\right)$ and the primary effect for speed was significant $\left(\mathrm{F}_{2}=1.35, \mathrm{P}=0.001\right)$. The interaction effect of bimanual modes and the pace was pronounced at $\left(\mathrm{F}_{2}=1.27, \mathrm{P}=0.001\right)$ (Table 2$)$. The two-way interactional significance for speed $\times$ sensory condition was presented to the Bonferroni Test for further analysis. The in-phase mode results indicated a pronouncement with the same level of consistency across the 3 differ-

Table 1. Results of two-way repeated measures ANOVA for the sensorimotor rhythm power at different speeds

\begin{tabular}{cccccc}
\hline Source & df & Mean & F & Sig. & Partial Eta Squared \\
\hline Bimanual patterns & 1 & 289.91 & 330.04 & 0.001 & 0.89 \\
Speed & 2 & 11.99 & 11.83 & 0.001 & 0.001 \\
Bimanual patterns* speed & 2 & 9.54 & 11.99 & 0.001 & \\
\hline
\end{tabular}

* Interaction effect 
Table 2. The results of two-way repeated measures ANOVA for the error of relative phase

\begin{tabular}{cccccc}
\hline Source & df & Mean & F & Sig. & Partial Eta Squared \\
\hline Bimanual patterns & 1 & 5026.004 & 7.390 & 0.001 & 0.99 \\
Speed & 2 & 1464.217 & 1.352 & 0.001 & 0.97 \\
Bimanual patterns* speed & 2 & 1503.762 & 1.273 & 0.001 \\
\hline
\end{tabular}

* Interaction effect

PHYSICAL TREA $\ M E N T S$

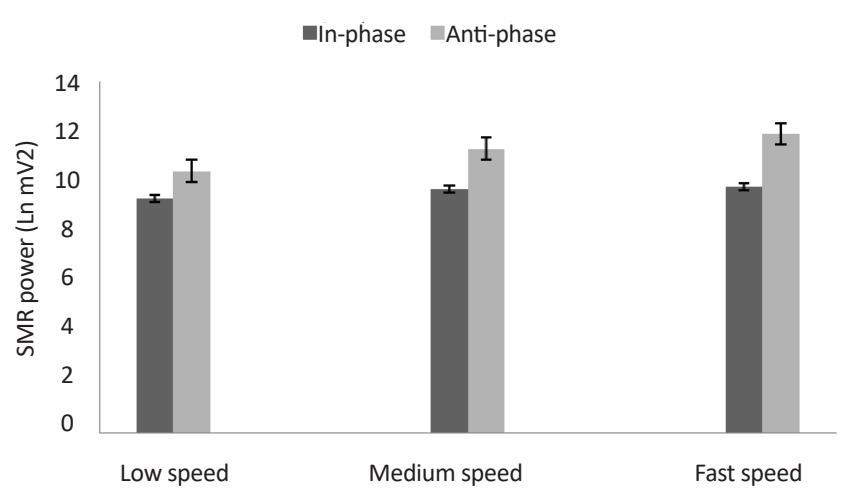

PHYSICAL TREA $\mid M E N T S$

Figure 1. The sensorimotor rhythm power in the in-phase and anti-phase bimanual coordination at different speeds

ent speeds. The anti-phase results, however, indicate that the effects of speed were significant for all $(\mathrm{P}=0.001)$. A notable significant increase of $\mathrm{AE}$ results on slow versus medium pace $(\mathrm{P}=0.001)$, slow versus fast pace $(\mathrm{P}=0.001)$ and medi um versus fast pace $(\mathrm{P}=0.001)$ were noticed through the pairwise comparison. These observations suggest that the increasing movement speed strongly influences the performance of anti-phase coordination model.

\section{Discussion}

Several strategies are available in which neurofeedback training proves to be a prominent example to enhance skills [30]. Moreover, a wealth of evidence from experimental, cross-sectional, and longitudinal studies suggests that the neurofeedback training is useful in the performance of athletes [31]. However, the role of neurofeedback in the performance of bimanual coordination and its impact on increased speed in the execution of the movement patterns is limited. There is no appropriate theory or prior research findings on the differences of the SMR power between in-phase and anti-phase patterns [32].

The status of this wave is not entirely understood. Therefore, the present study investigated the abovementioned issues. The experiment was designed to examine the SMR power during performances of the

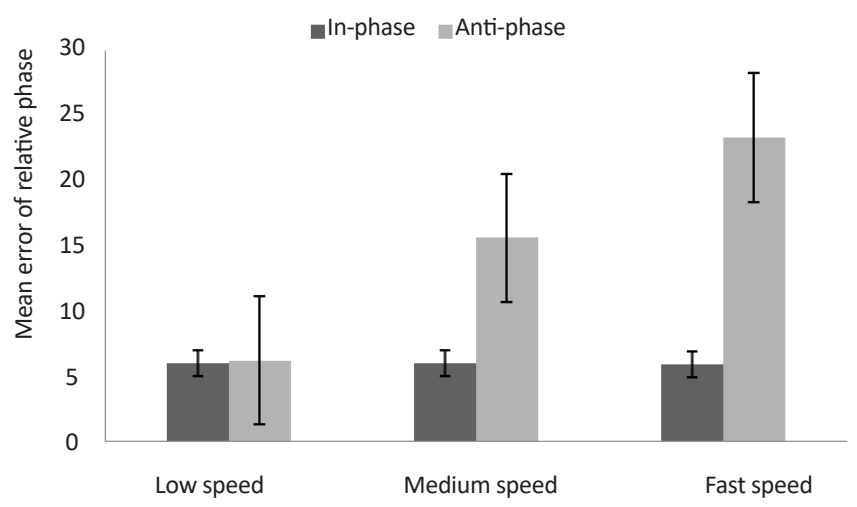

Figure 2. Significant main effects for bimanual coordination pattern 
2 fundamental phase patterns in the coordination of bimanual task at different speeds. Healthy individuals participated in this study. They were requested to use their hands (wrists) while EEG was recorded during the 2 opposing phase movements. The results suggested that SMR power was greater in the anti-phase setting, compared to the in-phase.

It is concluded that SMR may be an indicator of the attentive effort, which is intense in anti-phase pattern. However, it is insufficient to compensate for the disrupting constraints that hamper the anti-phase coupling. These results indicate that SMR power is essential for a skillful performance of the bimanual task. When the speed was increased, the study participants performed anti-phase coordination tasks with higher SMR power. In other words, the SMR power is an indicator of accurate performance.

These findings are mainly supported by observations from previous studies where SMR power was essential for performance enhancement [10, 11, 31, 33]. Furthermore, SMR is considered as an indicator for activating the cortex that related in the automatic process-related attention and skilled visuo-motor performance [34, 35]. Additionally, SMR power that is augmenting might alter the processes associated with attention. This process occurs through the enhancement of impulse control and the power to combine critical environmental stimuli [36].

According to the increased SMR activities, the sensorimotor cortex maintains the relaxed and focused states through reduction of the motor perception (e.g. somatosensory processing) [5]. This finding is consistent with the principle of automaticity mentioned previously [37]. Based on learning model of Fitz and Posner, abundant evidence suggests that peak performance is a sense of relaxation [37]. Thus, neurofeedback training can be used in several sports and exercise sessions to improve skills. These findings are consistent with Doppelmayr and Weber's conclusions [11]. They revealed that augmented SMR neurofeedback training results in a significant facilitation of spatial rotation task performance. The spatial rotation ability is necessary to perform specific bimanual tasks [11].

Neurofeedback is a tool for instruction and therapies in neurological procedures [38]. Studies suggest that applying neurofeedback training can convert abnormal brain rhythms and frequencies to the normal ones [39]. Neurofeedback has been formed based on the mind-body connection theory that includes training the brain to increase operating capabilities and optimal manner to experience optimal states of mind [40].

Part of this experimental investigation was to study the function of speed and coordination of movements. Increasing speed enhanced activity of the 1800 anti-phase model, but failed to affect the 00 in-phase model. With gradual increase in speed, the stable 1800 anti-phase became weak and less stable. This occurs with growing precision and decrease in flexibility. However, the design in the 00 in-phase did not change across various frequencies. This is a replication of the findings from earlier research $[12,21,22,28]$.

Considering the potential applications of this research, sports experts, psychologists and coaches can observe the results of this study in performance enhancements. If there is such an effect on SMR power and bimanual coordination task, we can adopt the best strategies for the development of these useful factors on motor performance and learning in bimanual coordination task. The most useful deduction from this research is that SMR neurofeedback training could enhance accuracy in bimanual coordination. The SMR neurofeedback training can be considered as a training protocol to improve bimanual coordination performance. However, applying a cross-sectional design was a limitation to our study. Future studies are recommended to examine the obtained results through longitudinal designs.

\section{Ethical Considerations}

\section{Compliance with ethical guidelines}

All procedures performed in studies involving human participants were in accordance with the ethical standards of the institutional and/or national research committee and with the 1964 Helsinki declaration and its later amendments or comparable ethical standards.

\section{Funding}

This research did not receive any specific grant from funding agencies in the public, commercial, or not-forprofit sectors.

\section{Authors contributions}

Conceptualization: Both authors; Methodology: Ebarhim Norouzi; Investigation: Both authors; Writing and Original Draft: Ebarhim Norouzi; Writing, Reviewing and Editing: Both authors; Funding Acquisition: 
Both authors; Resources: Both authors; Supervision: Mohammad Vaezmousavi.

\section{Conflict of interest}

The authors declared no conflict of interest.

\section{Acknowledgements}

We thank Milad Arabloo (Sharif University of Technology) for proofreading the manuscript.

\section{References}

[1] Gallicchio G, Cooke A, Ring C. Lower left temporal-frontal connectivity characterizes expert and accurate performance: High-alpha T7-Fz connectivity as a marker of conscious processing during movement. Sport, Exercise, and Performance Psychology. 2016; 5(1):14-31. [DOI:10.1037/spy0000055]

[2] Hatfield BD, Landers DM, Ray WJ. Cognitive processes during self-paced motor performance: An electroencephalographic profile of skilled marksmen. Journal of Sport Psychology. 1984; 6(1):42-59. [DOI:10.1123/jsp.6.1.42]

[3] Ishii R, Shinosaki $\mathrm{K}$, Ukai S, Inouye $\mathrm{T}$, Ishihara $\mathrm{T}$, Yoshimine T, et al. Medial prefrontal cortex generates frontal midline theta rhythm. Neuroreport. 1999; 10(4):675-9. [DOI:10.1097/00001756-199903170-00003] [PMID]

[4] Cheng MY, Hung CL, Huang CJ, Chang YK, Lo LC, Shen $\mathrm{C}$, et al. Expert-novice differences in SMR activity during dart throwing. Biological Psychology. 2015; 110(2):212-8. [DOI:10.1016/j.biopsycho.2015.08.003] [PMID]

[5] Vernon D, Egner T, Cooper N, Compton T, Neilands C, Sheri A, et al. The effect of training distinct neurofeedback protocols on aspects of cognitive performance. International Journal of Psychophysiology. 2003; 47(1):75-85. [DOI:10.1016/ S0167-8760(02)00091-0]

[6] Gruzelier J, Egner T, Vernon D. Validating the efficacy of neurofeedback for optimising performance. Progress in Brain Research. 2006; 159:421-31. [DOI:10.1016/S00796123(06)59027-2]

[7] Gruzelier JH. EEG-neurofeedback for optimising performance. I: A review of cognitive and affective outcome in healthy participants. Neuroscience \& Biobehavioral Reviews. 2014; 44(2):124-41. [DOI:10.1016/j.neubiorev.2013.09.015] [PMID]

[8] Chuang LY, Huang CJ, Hung TM. The differences in frontal midline theta power between successful and unsuccessful basketball free throws of elite basketball players. International Journal of Psychophysiology. 2013; 90(3):321-8 [DOI:10.1016/j.ijpsycho.2013.10.002] [PMID]

[9] Hosseini F, Norouzi E. Effect of neurofeedback training on self-talk and performance in elite and non-elite volleyball players. Medicina Dello Sport. 2017; 70(3):344-53. [DOI: 10.23736/S0025-7826.16.03011-8]
[10] Wilson VE, Peper E, Moss D. “The mind room" in Italian soccer training: The use of biofeedback and neurofeedback for optimum performance. Biofeedback. 2006; 34(3):79-81.

[11] Doppelmayr M, Weber E. Effects of SMR and theta/beta neurofeedback on reaction times, spatial abilities, and creativity. Journal of Neurotherapy. 2011; 15(2):115-29. [DOI:10.1 080/10874208.2011.570689]

[12] Kelso JA. Phase transitions and critical behavior in human bimanual coordination. American Journal of PhysiologyRegulatory, Integrative and Comparative Physiology. 1984; 246(6):R1000-4. [DOI:10.1152/ajpregu.1984.246.6.R1000] [PMID]

[13] Lee TD, Swinnen SP, Verschueren S. Relative phase alterations during bimanual skill acquisition. Journal of Motor Behavior. 1995; 27(3):263-74. [DOI:10.1080/00222895.1995.9 941716] [PMID]

[14] Swinnen SP, Jardin K, Meulenbroek R, Dounskaia N, Hofkens-Van Den Brandt M. Egocentric and allocentric constraints in the expression of patterns of interlimb coordination. Journal of Cognitive Neuroscience. 1997; 9(3):348-77. [DOI:10.1162/jocn.1997.9.3.348] [PMID]

[15] Temprado JJ, Chardenon A, Laurent M. Interplay of biomechanical and neuromuscular constraints on pattern stability and attentional demands in a bimanual coordination task in human subjects. Neuroscience Letters. 2001; 303(2):127-31. [DOI:10.1016/S0304-3940(01)01650-0]

[16] Swinnen SP. Intermanual coordination: from behavioural principles to neural-network interactions. Nature Reviews. Neuroscience. 2002; 3(5):348-59. [DOI:10.1038/nrn807] [PMID]

[17] Norouzi E, Farsi A, Vaezmousavi M. Effects of proprioceptive and visual disturbance on inphase and anti-phase hand performance. Physical Treatments-Specific Physical Therapy Journal. 2015; 5(1):41-8.

[18] Salter JE, Wishart LR, Lee TD, Simon D. Perceptual and motor contributions to bimanual coordination. Neuroscience Letters. 2004; 363(2):102-7. [DOI:10.1016/j.neut1 let.2004.03.071] [PMID]

[19] Baldissera F, Cavallari P, Marini G, Tassone G. Differential control of in-phase and anti-phase coupling of rhythmic movements of ipsilateral hand and foot. Experimental Brain Research. 1991; 83(2):375-80. [DOI:10.1007/BF00231161] [PMID]

[20] Swinnen SP, Dounskaia N, Verschueren S, Serrien DJ, Daelman A. Relative phase destabilization during interlimb coordination: The disruptive role of kinesthetic afferences induced by passive movement. Experimental Brain Research. 1990; 105(3):439-54. [DOI:10.1007/BF00233044]

[21] Kelso JA, Jeka JJ. Symmetry breaking dynamics of human multilimb coordination. Journal of Experimental Psychology: Human Perception and Performance. 1992; 18(3):645-68. [DOI:10.1037/0096-1523.18.3.645] [PMID]

[22] Li Y, Levin O, Carson RG, Swinnen SP. Bimanual coordination: Constraints imposed by the relative timing of homologous muscle activation. Experimental Brain Research. 2004; 156(1):27-38. [DOI:10.1007/s00221-003-1762-4] [PMID]

[23] Li Y, Levin O, Forner-Cordero A, Ronsse R, Swinnen SP. Coordination of complex bimanual multijoint movements under increasing cycling frequencies: The prevalence of 
mirror-image and translational symmetry. Acta Psychologica. 2009; 130(3):183-95. [DOI:10.1016/j.actpsy.2008.12.003] [PMID]

[24] Scholz JP, Kelso JA. Intentional switching between patterns of bimanual coordination depends on the intrinsic dynamics of the patterns. Journal of Motor Behavior. 1990; 22(1):98124. [DOI:10.1080/00222895.1990.10735504]

[25] Doppelmayr M, Klimesch W, Pachinger T, Ripper B. Individual differences in brain dynamics: Important implications for the calculation of event-related band power. Biological Cybernetics. 1998; 79(1):49-57. [DOI:10.1007/ s004220050457] [PMID]

[26] Zaidel E, Barnea A. Symposium IV: Quantitative EEG and neurofeedback. Brain and Cognition. 2006; 60(3):329-30. [DOI:10.1016/j.bandc.2005.09.004]

[27] Oldfield RC. The assessment and analysis of handedness: The Edinburgh inventory. Neuropsychologia. 1971; 9(1):97113. [DOI:10.1016/0028-3932(71)90067-4]

[28] Grillo EU, Almeida Q, Lee TD, Abbott KV. Do vision and audition influence bimanual timing coordination for inphase and anti-phase patterns in a linear slide task. The Open Sports Sciences Journal. 2010; 3:105-10. [DOI:10.2174/ 1875399X010030100105]

[29] Chatrian GE, Lettich E, Nelson PL. Ten percent electrode system for topographic studies of spontaneous and evoked EEG activities. American Journal of EEG technology. 1985; 25(2):83-92. [DOI:10.1080/00029238.1985.11080163]

[30] Strack B, Linden M, Wilson VS. Biofeedback \& neurofeedback applications in sport psychology. Wheat Ridge, Colorado: Association for Applied Psychophysiology and Biofeedback; 2011.

[31] Rostami R, Sadeghi H, Karami KA, Abadi MN, Salamati $P$. The effects of neurofeedback on the improvement of rifle shooters' performance. Journal of Neurotherapy. 2012; 16(4):264-9. [DOI:10.1080/10874208.2012.730388]

[32] Sherlin LH, Larson NC, Sherlin RM. Developing a Performance Brain Training ${ }^{\mathrm{TM}}$ Approach for Baseball: A Process Analysis with Descriptive Data. Applied Psychophysiology and Biofeedback. 2013; 38(1):29-44. [DOI:10.1007/s10484012-9205-2] [PMID]

[33] Casey M, Yau A, Barfoot K, Callaway A. Data mining of portable EEG brain wave signals for sports performance analysis: An archery case study. Paper presented at: The International Conference on Science, Education and Medicine in Sport. 19-24 July 2012; Glasgow, Scotland.

[34] Ring C, Cooke A, Kavussanu M, McIntyre D, Masters R. Investigating the efficacy of neurofeedback training for expediting expertise and excellence in sport. Psychology of Sport and Exercise. 2015; 16:118-27. [DOI:10.1016/j.psychh sport.2014.08.005]

[35] Mann CA, Sterman MB, Kaiser DA. Suppression of EEG rhythmic frequencies during somato-motor and visuomotor behavior. International Journal of Psychophysiology. 1996; 23(1):1-7. [DOI:10.1016/0167-8760(96)00036-0]

[36] Egner T, Zech TF, Gruzelier JH. The effects of neurofeedback training on the spectral topography of the electroencephalogram. Clinical Neurophysiology. 2004; 115(11):245260. [DOI:10.1016/j.clinph.2004.05.033] [PMID]
[37] Fitts PM, Posner MI. Human performance. Washington: American Psychological Association; 1967.

[38] Sherwood MS, Diller EE, Ey E, Ganapathy S, Nelson JT, Parker JG. A protocol for the Administration of Real-Time fMRI Neurofeedback Training. Journal of Visualized Experiments. 2017; (126):55543. [DOI:10.3791/55543] [PMID] [PMCID]

[39] Blumenstein B, Bar-Eli M, Tenenbaum G. Brain and body in sport and exercise: Biofeedback applications in performance enhancement. Hoboken, New Jersey: John Wiley \& Sons; 2002.

[40] Acevedo EO, Ekkekakis P. Psychobiology of physical activity. Champaigns: Human Kinetics; 2006. 